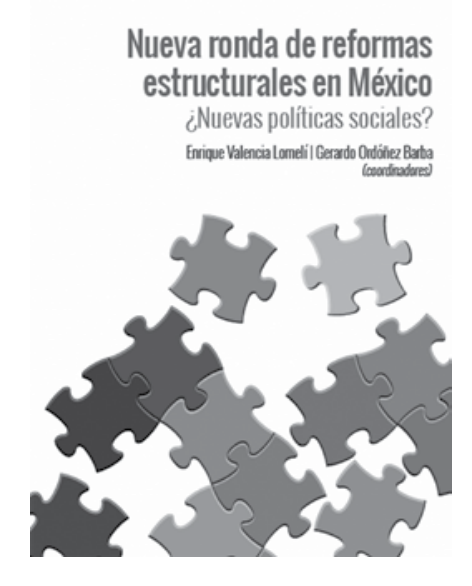

Nueva ronda de reformas

icturales en México Nuevas políticas sociales? Coovidnaderes

- Nueva ronda de reformas estructurales

en México. ¿Nuevas políticas sociales?

ENRIQUe VAlencia LOMELÍ

y GERARDO ORDÓNEEZ BARBA, 2016

El Colegio de la Frontera Norte, México

\section{Reformas sin ronda: la narrativa triunfalista del mercado}

\author{
JAIME ANTONIO PRECIADO CORONADO
}

Reforms without a Round: The Market's Triumphalist

Narrative

Jaime Antonio Preciado Coronado

Universidad de Guadalajara, Guadalajara, Jalisco, México japreco@hotmail.com

Desacatos 56

enero-abril 2018, pp. 202-207
En definitiva, las nuevas rondas de reformas que encadenan la dimensión social a la reforma del mercado no atacan a fondo el añejo n una economía marcada por criterios de mercado, las reformas llamadas estructurales han acentuado la estabilidad macroeconómica y relegado las políticas sociales a un papel adaptativo, frente a una crisis global y sistémica del ciclo actual de la acumulación capitalista. En un trabajo conjunto entre el Instituto para la Integración de América Latina y el Caribe (Intal), el Banco Interamericano de Desarrollo (BID) y Corporación Latinobarómetro, se señala que:

En la última década proliferaron programas sociales de asistencia, se multiplicó la clase media y se expandió el acceso a servicios básicos de educación y salud. Sin embargo, 175 millones de personas aún viven en la pobreza y persisten desigualdades en materia de ingresos y oportunidades [...], el 55\% del empleo es informal o precario [...]. La brecha de ingresos entre trabajadores formales e informales se acrecienta cuando alcanzan la edad jubilatoria y [...] sólo la mitad de la población activa ahorra para el momento de su jubilación (Beliz y Chelala, 2016: 78). 
problema de la desigualdad, donde está el corazón de la cuestión social.

A la par de una percepción pública, que hace ver como intolerables los polarizados indicadores de la desigualdad, hay una narrativa triunfalista de las reformas de mercado, al suponer que las "mejoras" económicas llevan de manera automática a las "mejoras sociales". Sin embargo, la desigualdad social sigue generando descontento y fragmentación entre los ciudadanos, impide su cohesión social y produce desestabilización política. La democracia se debilita y no parece que las reformas sociales tiendan a fortalecerla. Entre 1990 y 2015, de acuerdo con un estudio de Latinobarómetro (2016), creció la desconfianza en Latinoamérica, marcada por la desigualdad y por una sociedad que funciona en redes que están interconectadas débilmente dentro de la sociedad. Rosanvallon (2016) señala que:

La democracia trajo consigo la aspiración de igualdad ante la ley, la aspiración a la participación con capacidad de decisión, es decir la soberanía del voto, y por cierto trajo la aspiración de las garantías sociales además de muchas otras [...]. Este conjunto de aspiraciones, junto con el sufragio universal de todos, produjo democracias electorales, que en muchos casos son semisoberanas, porque la soberanía no reside totalmente en la gente.

En la primera parte del libro, "Nuevas reformas estructurales: narrativas agotadas", se parte de un enfoque general que incluye cinco textos. En los tres primeros, se discute el contexto económico y político de las nuevas reformas. Enrique Valencia Lomelí escribe "Viejas y recientes reformas: trayectoria de un mismo ciclo". Sitúa una línea de continuidad en torno a las narrativas históricas vinculadas a las reformas "estructurales" emprendidas por Enrique Peña Nieto en México y destaca el triunfalismo de la ortodoxia neoliberal. En su capítulo, "Para ir más allá de la pobreza: una reforma macroeconómica del Estado para el desarrollo", Rolando Cordera Campos plantea un debate sobre otra manera de enfrentar la política económica mediante la diversificación y profundización de la actividad productiva y la constitución de plataformas institucionales y de fomento productivo, en un desarrollo con objetivos de inclusión social, empleo y abatimiento de la pobreza y de carencias básicas. En una visión amplia y detallada del marco político reciente, Alberto Aziz Nassif, en "Grandes expectativas, pobres resultados: el regreso del PRI y el mito de las reformas", propone recuperar la capacidad reguladora del Estado mexicano para hacer gobernable al país, la reconfiguración del presidencialismo con capacidades de gobernar mediante pactos sociales no partidocráticos y una coalición reformista capaz de gobernar sin mayoría por medio de un pacto político.

Los dos siguientes capítulos de esta parte se enfocan en las implicaciones de las reformas en estrategias e instituciones sociales en general. Carlos Barba Solano analiza "Las propuestas de reformas y las nuevas estrategias sociales del gobierno de Enrique Peña Nieto. ¿Cambio de rumbo en la política social mexicana?". En particular, se enfoca en las nuevas estrategias de política social, como la Cruzada Nacional contra el Hambre y algunas reformas constitucionales en materia de derechos sociales, como la Ley de la Pensión Universal y la Ley del Seguro de Desempleo, acciones públicas interpretadas como factores que impulsarán cambios significativos y progresivos en el desarrollo de la política social en México, cuyo discurso busca universalizar el acceso a los sistemas de protección social. Luis Ignacio Román Morales, en "Las reformas estructurales y el empleo: de la prioridad discursiva al desdén efectivo", constata que la pérdida de poder adquisitivo de los ingresos, la precarización laboral, la pérdida de posibilidades reales para exigir derechos, las jornadas extenuantes, la recurrencia a la migración, la economía informal, 
la ilegalidad o la exclusión de la participación económica — los ninis_, ${ }^{1}$ abarcan proporciones crecientes de la población sin que haya políticas pertinentes para el empleo.

La segunda parte de esta obra, "Enfoques históricos y sociales de la reforma tributaria", está integrada por tres artículos relacionados con la reforma fiscal de Enrique Peña Nieto y sus impactos sociales. Con un enfoque histórico y comparativo, Mónica Unda Gutiérrez ofrece el capítulo "El sistema tributario mexicano en perspectiva histórica e internacional después de la reforma de 2013". Analiza el papel del Ejecutivo y el Legislativo en el proceso de reforma a las leyes de los impuestos principales del sistema tributario, y muestra su trayectoria en las últimas décadas en comparación con los sistemas tributarios de otros países. Concluye que la reforma es insuficiente para atajar un problema grave, añejo y muy distintivo del caso mexicano. También desde una perspectiva histórica, Gerardo Ordóñez Barba, en "Las finanzas públicas y el gasto social en las últimas tres décadas en México, 1982-2014”, describe la evolución de las finanzas públicas federales, con sus principales debilidades y la manera en que han afectado la capacidad del Estado para financiar el gasto nacional, en particular las partidas del presupuesto destinadas al desarrollo social. Cierra esta parte un capítulo de enfoque econométrico. Luis Huesca Reynoso, Arturo Robles Valencia y Abdelkrim Araar hacen una "Medición generalizada del efecto redistributivo del sistema fiscal en México: ¿sin recaudar y sin redistribuir?", que analiza los estudios de progresividad y el efecto redistributivo de las transferencias o impuestos en los hogares, pobres y no pobres. Se preguntan si su aplicación es adecuada y si las vías de reforma provocan que su impacto en la población sea el mejor. Aportan un conocimiento de primera mano sobre quiénes — estratos-y en qué medida - recursosse benefician de la política fiscal en general.

En cuatro textos vinculados a las reformas sociales de Peña Nieto, la tercera parte de este libro se pregunta: “¿Hacia una nueva generación de reformas en la seguridad social y la salud?". En el trabajo "La transformación de la seguridad social. ¿Hacia un modelo de subsistencia?”, Manuel Canto Chac y Tania Garduño Nando lanzan la hipótesis de que en toda Latinoamérica se perciben transiciones en la política y en la seguridad social, impulsadas por los cambios en su estructura demográfica. En México, esta transición está orientada por la sustitución de derechos, reemplazados por un sistema cuasi universalizado de servicios básicos. Para demostrarlo, confrontan la propuesta presidencial de Peña Nieto con la articulada por Santiago Levy en torno al Instituto Mexicano del Seguro Social. Para Graciela Bensusán, su colaboración "Aciertos, desaciertos y posibles mejoras en el diseño del seguro de desempleo en México" deja ver que los posibles seguro y pensión universal fueron parte de una iniciativa de reforma hacendaria y seguridad social propuesta por el poder Ejecutivo en 2014, que tuvo un propósito legitimador de la reforma tributaria, porque la recaudación permitiría avanzar hacia un "sistema de seguridad social universal” y dejaría atrás una "política social asistencialista". Sin embargo, la reforma no avanzó en esa dirección, sino que, "por su esquema de financiamiento, basado en parte de las aportaciones patronales destinadas anteriormente al acceso a un crédito de vivienda por parte de los trabajadores, generó el rechazo de amplios sectores de la sociedad" (p. 331). En el siguiente capítulo, Berenice P. Ramírez López también se refiere a "La seguridad social y la pensión universal”. Recomienda que, ante la debilidad del gobierno federal, éste se

“El término 'nini' se utiliza para definir a aquellos jóvenes de entre 14 y 29 años que por diversos factores ni estudian, ni trabajan. Según datos de la Universidad Nacional Autónoma de México (UNAM) existen alrededor de siete millones, y aunque no es un dato exacto sí resulta preocupante". Véase <https://www.monografias.com/docs/Ori gen-de-los-ninis-PK5QQSYBZ>. 
proponga una pensión no contributiva, pues habría que reconocer que la cobertura de la seguridad social es insuficiente. Contrasta que en Latinoamérica 14 países cuentan con pensiones no contributivas de financiamiento público y que en sus inicios fue focalizada la población vulnerable y en extrema pobreza, mientras que cinco países han adoptado la forma de pensiones solidarias, básicas o sociales.

Esta parte termina con el capítulo de Oliva López Arellano y José Alberto Rivera Márquez, "Sistema Nacional de Salud Universal: universalidad de mercado y restricción de derechos". Para ellos, la política neoliberal en salud modela el sistema sanitario mediante la reconfiguración del financiamiento, la privatización de lo público rentable, la selectividad en las intervenciones y la focalización de poblaciones. Así, se mercantilizan de manera gradual las instituciones públicas, se fortalecen los discursos tecnocráticos en detrimento de lo político, y se diluye la responsabilidad del Estado como "el sujeto obligado de garantizar los derechos humanos, trasladando a los individuos la tarea de gestionar/administrar sus riesgos para mejorar su salud" (p. 388).

La cuarta parte, "Reformas recientes en las acciones públicas frente a la pobreza y la vulnerabilidad", incluye dos trabajos relacionados con la Cruzada Nacional contra el Hambre. En el primero, de Iliana Yaschine Arroyo, Sara Ochoa León y Citlalli Hernández Juárez, "Cruzada Nacional Contra el Hambre: análisis de su diseño desde un enfoque de seguridad alimentaria", se examina el diseño de la Cruzada como una estrategia para garantizar la seguridad alimentaria de la población con base en el enfoque de seguridad alimentaria de la Organización de las Naciones Unidas para la Alimentación y la Agricultura (FAO), sus objetivos, su consistencia interna, así como la alineación de sus instrumentos de intervención. Julio Boltvinik Kalinka, en "Conceptos y mediciones de pobreza y políticas públicas. La medición multidimensional del Coneval y la Cruzada Nacional Contra el Hambre", analiza la influencia directa del Método de Medición Multidimensional Oficial de la Pobreza (MмMOP) del Consejo Nacional de Evaluación de la Política de Desarrollo Social (Coneval), en el diseño de la Cruzada. Destaca la falta de claridad conceptual reinante al respecto, en particular en México, y las razones por las que las mediciones usuales subestiman la incidencia social de estos problemas. Da un panorama de las mediciones de desnutrición, pobreza alimentaria e inseguridad alimentaria que imperan en el país y las compara con una medición de pobreza alimentaria alternativa a la tradicional. Desmenuza de manera crítica — deconstruye - la conceptualización y metodología utilizadas, que desembocan en una lectura mecánica, pues la Cruzada terminó justificando la definición "ultraminimalista" de su población objetivo, con lo que dejó a millones de personas fuera.

Esta parte incluye un debate planteado por Araceli Damián González, "Constelaciones de poder, los intelectuales y la transformación del Estado de bienestar y la política social en México”, que analiza las recientes transformaciones del Estado de bienestar y la política social en México, respecto al papel de los intelectuales en su reconfiguración, lo que lleva a profundizar los principios neoliberales en materia social. Critica también la medición oficial de la pobreza, en tanto "refleja el ejercicio arbitrario en el establecimiento de normas sociales" (p. 502). Desde la perspectiva ambiental, el trabajo de Rodrigo Flores Elizondo, "Programa contra la sequía: entre la construcción de resiliencia y la construcción de vulnerabilidad social", plantea que los horizontes de racionamiento de aguas "pueden llegar a ser caóticos y desequilibrantes de la calidad de vida de los usuarios y de la certidumbre económica de las regiones si no se planean con tiempo. Si de lo que se trata es de reducir la vulnerabilidad, la respuesta tendría que ser de construcción de resiliencia hídrica" (p. 537).

La quinta parte, "Reformas estructurales: ¿hacia una nueva institucionalidad?”, incluye tres

Reformas sin ronda: la narrativa triunfalista del mercado 
trabajos de corte neoinstitucionalista. Empieza con el texto de Alfonso Celestino Pérez, "Los derechos humanos en las instituciones públicas. ¿Una nueva generación de instituciones para el bienestar?”, que parte del estudio de diagnóstico y evaluación realizado por un grupo de investigadores de Iniciativa Ciudadana y Desarrollo Social (Incide Social), durante 2013. Se valora si el enfoque de derechos impacta a los denominados "entes implementadores de institucionalidad pública" (p. 570) de la Ciudad de México, así como las líneas de acción del Programa de Derechos Humanos del Distrito Federal. Sus conclusiones se dirigen al gobierno federal como retos y áreas susceptibles de mejora para la administración pública nacional en su conjunto. Israel Banegas González y Tania Hernández ofrecen el texto "Sociedad civil y desarrollo social: ¿qué podemos esperar de la nueva administración?”, como un esbozo de las continuidades y divergencias en la política de fomento a las organizaciones de la sociedad civil (OSC), por parte de la administración federal de Peña Nieto. Se revisan las acciones de las dos gestiones anteriores y los dos primeros años de la actual para identificar los temas de desarrollo social propuestos por las OSC y las formas en que éstas se han relacionado con el gobierno federal. Esta quinta parte finaliza con el estudio de Gonzalo Hernández Licona sobre "Autonomía de hecho y autonomía de derecho: los retos de la reforma política para el Coneval", que evalúa el potencial de las políticas sociales en el marco de la Ley General de Desarrollo Social, dentro de la cual el Coneval ha contado con autonomía en los hechos, que proviene de varias fuentes, pero en especial de la estructura interna que el propio Congreso le otorgó y del desempeño apartidista de sus integrantes. Autonomía, sin embargo, amenazada desde finales de 2015, junto con la del Instituto Nacional de Estadística y Geografía.

La sexta parte, "Reformas ausentes: las políticas del cuidado", examina de manera pertinente la relación entre la estructura etaria y la política social. Rocío Enríquez Rosas y David Foust Rodríguez presentan, en "Envejecimiento, redes y protección social en México: reflexiones a partir de estudios de caso", la intersección de las emociones y las redes sociales como lugar testimonial de carencias del sistema de protección social en los ámbitos del cuidado a los ancianos y las pensiones. Dan testimonio de la agencia de las personas mayores y de sus familias dentro de la solidaridad desplegada como recurso —erosionado o en proceso de estarlo- “en un régimen de bienestar que propende al residualismo y el familiarismo" (p. 665), lo cual aleja reformas sustantivas en el campo del cuidado de los adultos mayores. En la otra punta demográfica, Óscar Alfonso Martínez Martínez, Adolfo Rogelio Cogco Calderón y Jorge Alberto Pérez Cruz tratan la "Satisfacción de los beneficiarios del Programa de Estancias Infantiles: evaluación de la política social del gobierno federal en México". La dinámica familiar cambia con la incorporación de la mujer a las actividades económicas, incluso en áreas casi exclusivas de los varones. Ello no significa que las actividades domésticas se redistribuyan en el interior del hogar de manera equitativa. Aparecen así los servicios de cuidado de niños, más allá de la familia, por lo regular de las mujeres, en establecimientos particulares de cobro. Esto es un problema para las unidades domésticas en pobreza, con mujeres que optan por no trabajar de manera remunerada fuera del hogar. Sin embargo, el programa analizado es insuficiente para atender la creciente demanda de atención pública infantil.

Las seis partes en las que se divide este libro plantean la pregunta de cómo las reformas económicas, políticas y sociales pueden crear una sociedad de iguales. Rosanvallon (2016) ofrece algunas pistas:

En estos días, la igualdad suele definirse matemáticamente, como una comparación de las posiciones económicas de individuos o segmentos 
de la población. Esta noción tiene sus usos. Pero la igualdad debe también, y tal vez principalmente, ser definida socialmente, como una medida del vínculo comunal [...].

Una teoría de la igualdad debe centrarse en la estructura de la sociedad. Debe descansar en tres principios: el reconocimiento de la singularidad de las personas (en contraposición al individualismo), la organización de la reciprocidad (en la relación de los ciudadanos entre sí) y la constitución de la comunidad (para la comunidad en su conjunto).
Hacia esos espacios de incertidumbre, pero también hacia un reforzamiento de la capacidad de agencia humana, nos llevan las seis partes del libro que comentamos, pues a pesar de que las opciones liberales o populistas han mostrado severas limitaciones, la capacidad crítica que impulsa la Red Mexicana de Investigación en Política Social (Remipso), espacio en el que convergen los autores que contribuyen a esta obra, aporta nuevas sinergias entre academia, políticas públicas y acciones colectivas fundadoras de nuevas condiciones para la equidad social. D

\section{Bibliografía}

Beliz, Gustavo y Santiago Chelala, 2016, El ADN de la integración regional: la voz de los latinoamericanos por una convergencia de calidad: innovación, equidad y cuidado ambiental, Banco Interamericano de Desarrollo/Instituto para la Integración de América Latina y el Caribe.

Corporación Latinobarómetro, 2016, La Confianza en América Latina 1995-2015. 20 años de opinión pública latinoamericana, Corporación Latinobarómetro, Santiago. Disponible en línea: <https://www.google.com.mx/url?sa=t\&rct=j\&q=\&esrc=s\&source=web\&cd=1\&ved=0a hUKEwiiu9Wu-_3VAhXn8YMKHRqRCusQFggmMAA\&url=http\%3A\%2F\%2Fwww.latinobarometro.org\%2FLATDocs\%2FF00005085INFORME_LB_LA_CONFIANZA_1995_2015.pdf\&usg=AFQjCNH164OF_zORIw9xhCUjbcXVJQn4cg>.

Rosanvallon, Pierre, 2016, "How to Create a Society of Equals. Overcoming Today's Crisis of Inequality", en Foreign Affairs, enero-febrero. Disponible en línea: <https://www.foreignaffairs.com/articles/2015-12-14/how-creae-society-equals>. 